\title{
Targeting cholesterol at different levels in the mevalonate pathway protects fatty liver against ischemia-reperfusion injury.
}

Laura Llacuna $^{1,2}$, Anna Fernández ${ }^{1,2}$, Claudia Von Monfort ${ }^{1,2}$, Núria Matías ${ }^{1,2}$, Laura Martínez ${ }^{1,2}$, Francisco Caballero ${ }^{1,2}$, Antoni Rimola ${ }^{2}$, Montserrat Elena ${ }^{3}$, Albert Morales ${ }^{1,2 *}$ José C. Fernández-Checa ${ }^{1,2,4^{*}}$ and Carmen García-Ruiz ${ }^{1,2 *}$.

${ }^{1}$ Liver Unit and Centro de Investigaciones Biomédicas Esther Koplowitz, IMDiM, Hospital Clínic i Provincial and CIBEREHD, IDIBAPS, and ${ }^{2}$ Department of Cell Death and Proliferation, Instituto Investigaciones Biomédicas de Barcelona, Consejo Superior de Investigaciones Científicas, 08036-Barcelona, Spain, ${ }^{3}$ Centro Diagnóstico Biomédico, Hospital Clinic, 08036-Barcelona, Spain, ${ }^{4}$ Research Center for Alcoholic Liver and Pancreatic Diseases, Keck School of Medicine of the University of Southern California, Los Angeles, CA, USA.

Running title: Cholesterol and hepatic ischemia-reperfusion injury.

Key words: Hepatic steatosis; ischemia/reperfusion; liver transplantation; triglycerides; cholesterol; statins; squalene synthase.

Abbreviations used: CD, choline-deficient diet; DCF, 2'-7'-dichlorofluorescein; FFA, free fatty acids; HC, hypercholesterolemic diet; HMGCoA R, hydroxymethylglutary CoA reductase; I/R, ischemia/reperfusion; LDL, low density lipoprotein; MDA, malondialdehyde; mitochondrial permeability transition, MPT; SAM, S-adenosyl-L- 
methionine; StAR, steroidogenic acute regulatory protein; TMRE, tetramethylrhodamine ethyl ester; TG, triglycerides; VLDL, very low density lipoprotein.

Conflict of interest: Nothing to report.

Corresponding authors: J.C. Fernandez-Checa, checa229@yahoo.com, or Albert Morales, amorales@clinic.ub.es, or Carmen Garcia-Ruiz, cgrbam@iiibb.csic.es. IIBB-CSIC, C/Rosello 161, 08036-Barcelona, Spain.

*AM, JC F-Ch and CGR share senior authorship. 


\section{ABSTRACT}

Background\&Aims: Liver steatosis enhances ischemia/reperfusion (I/R) injury and is considered a primary factor in graft failure after liver transplantation. Although previous reports have shown a role for qualitative steatosis (macrovesicular vs. microvesicular) in hepatic I/R injury, no studies have compared side by side the specific contribution of individual lipids accumulating in fatty liver to $\mathrm{I} / \mathrm{R}$ damage. Methods: We used nutritional and genetic models of micro and macrovesicular fatty livers exhibiting specific lipid profiles to assess their susceptibility to normothermic I/R injury. Results: Unlike choline-deficient (CD) diet-fed mice, characterized by predominant liver triglycerides/free fatty acids (TG/FFA) accumulation, mice fed a cholesterol-enriched (HC) diet, which exhibited enhanced hepatic cholesterol loading in mitochondria, were highly sensitive to I/R-induced liver injury. In vivo two-photon confocal imaging revealed enhanced mitochondrial depolarization and generation of reactive oxygen species following hepatic $\mathrm{I} / \mathrm{R}$ in $\mathrm{HC}$-fed but not in $\mathrm{CD}$-fed mice, consistent with decreased mitochondrial GSH (mGSH) observed in HC-fed mice. Moreover, ob/ob mice, characterized by increased hepatic TG, FFA and cholesterol levels, were as sensitive to I/R-mediated liver injury as mice fed the $\mathrm{HC}$ diet. Livers from ob/ob mice displayed increased StAR expression and mitochondrial cholesterol accumulation, resulting in mGSH depletion. Interestingly, atorvastatin therapy or squalene synthase inhibition in vivo attenuated StAR overexpression, mitochondrial cholesterol loading and mGSH depletion, protecting ob/ob mice from I/R-mediated liver injury. Conclusion: cholesterol accumulation, particularly in mitochondria, sensitizes to hepatic I/R injury, emerging as a novel target to prevent the enhanced damage of steatotic livers to I/R-mediated damage. 


\section{INTRODUCTION}

Liver transplantation is an effective treatment for chronic end-stage liver disease and acute liver failure. The decreasing number or quality of organs available for transplantation represents a serious hurdle for this therapy, leading to the use of suboptimal-quality grafts, such as steatotic livers, which may compromise the overall success of liver transplantation [1]. Due to the rising prevalence of obesity and type II diabetes among the general population, fatty livers constitute an expanding pool of marginal grafts available for transplantation. Hepatic steatosis is known to impose an additional risk of primary graft dysfunction [1]. Fat accumulation within hepatocytes increases cell volume resulting in a decreased sinusoidal space and impaired microcirculatory blood flow [2]. Nevertheless, the molecular underlying mechanisms the susceptibility of fatty livers to ischemia/reperfusion (I/R) injury remain incompletely understood.

Based on the morphology, size and percentage of hepatocytes exhibiting cytoplasmic fat deposits, it has been previously reported that fatty livers with macrovesicular steatosis are more susceptible to $\mathrm{I} / \mathrm{R}$ injury than those with microsteatosis, correlating with impaired hepatic perfusion and portal vein flow [3, 4]. However, the contribution of microvesicular steatosis to graft dysfunction after transplantation remains unclear. For instance, while some studies showed that livers with moderate microsteatosis are nonfunctional after transplantation [5], other reports discarded a role for microvesicular steatosis on graft or patient survival $[6,7]$. Hence, a better understanding of the mechanisms involved in the susceptibility of fatty livers to I/R injury may increase the availability of donor organs, providing a better chance for liver transplantation.

Hepatic steatosis is characterized by the accumulation of a heterogeneous population of lipids, predominantly triglycerides (TG), free fatty acids (FFA) and cholesterol 
(esterified and unsterified) [8]. While hepatic FFA and cholesterol can derive from adipose tissue or LDL uptake from the circulation, they can be synthesized de novo in the endoplasmic reticulum by pathways controlled at the transcriptional level by transcription factors SREBP-1c and SREBP-2, respectively [8, 9]. Triglycerides are synthesized from FFA and can be stored in lipid droplets or packed as VLDL particles for export to extrahepatic organs. Although previous studies have addressed the impact of qualitative steatosis (macrovesicular vs. microvesicular) to hepatic I/R injury [3-7], no studies have compared side-by-side the specific contribution of the individual lipids accumulating in fatty liver to I/R injury. In order to discern whether the type rather than the amount of fat contributes to the susceptibility of fatty livers to I/R injury, we used nutritional models of hepatic steatosis characterized by predominant TG/FFA or cholesterol accumulation by feeding a diet deficient in choline or enriched in cholesterol, respectively [10]. In addition, we used ob/ob mice, a genetic model of hepatic macrovesicular steatosis and cholesterol accumulation, to analyze the influence of cholesterol modulation by blocking hydroxymethylglutaryl CoA reductase (HMGCoAR), the regulatory enzyme in the mevalonate pathway which is transcriptionally controlled by SREBP-2, or by inhibition of squalene synthase, which catalyzes the first committed step towards cholesterol synthesis [11]. Our data indicate that cholesterol and its trafficking to mitochondria critically contributes to the susceptibility of fatty liver to I/R injury and that its targeting may represent a previously unrecognized opportunity for a better option in liver transplantation. 


\section{MATERIALS AND METHODS}

\section{Animals, diets and treatments.}

Male C57BL/6 mice were purchased from Charles River Laboratories (Wilmington, MA). $\underline{\mathrm{Ob} / \mathrm{Ob}}$ mice (in the $\mathrm{C} 57 \mathrm{BL} / 6$ background) were obtained from The Jackson Laboratories (Bar Harbor, Maine). $\underline{8-10 \text { weeks old mice (the number of mice per group }}$ is detailed in the corresponding figure legend) were housed under specific pathogen-free conditions with controlled temperature and humidity on a $12 \mathrm{hr}$ light-dark cycle. Mice were fed either a choline-deficient (CD) methionine sufficient diet or a sodium cholatesupplemented hypercholesterolemic $(2 \%, \mathrm{HC})$ diet for 2 days (Dyets Inc, Bethlehem, PA) as described previously [10]. Control mice received the standard chow pellets. $\mathrm{Ob} / \mathrm{ob}$ mice were dosed daily by oral gavage with atorvastatin (Pfizer) $(10 \mathrm{mg} / \mathrm{kg})$, the squalene synthase inhibitor YM-53601 (Sigma) $(15 \mathrm{mg} / \mathrm{kg}$ ) or vehicle 7 days before being subjected to partial normothermic I/R. The experimental animal protocol was approved by the Institut d'Investigacions Biomèdiques Agustí Pi I Sunyer (IDIBAPS) Animal Care and Use Committee.

\section{Partial hepatic ischemia.}

Male wild-type mice were anesthesized using a vaporizer system to deliver isoflurane/O2 inhalation. After a midline laparotomy, hepatic inflow to the median and left lobes was occluded by application of a micro-vascular clamp (Biemer clip, 0.29$\underline{0.39 \mathrm{~N}) \text { for } 60-90 \mathrm{~min} \text { as described previously }[12,13] \text { to prevent hepatic blood flow for }}$ 90 minutes. Mesenteric venous congestion was prevented by portal decompression through the right and caudate lobes. Blood samples and liver biopsies were taken at different periods after reperfusion for further evaluation, typically 4-6 hours postreperfusion when massive liver injur is observed $[12,13]$. Control animals were sham operated. 


\section{Mitochondria isolation.}

In some cases, livers after reperfusion were collected and processed for mitochondrial isolation by Percoll density gradient centrifugation, checking for mitochondrial purity and cross contamination with extramitochondrial compartments, as described previously $[10,14]$.

\section{In vivo two-photon confocal imaging.}

Hepatic partial warm ischemia was performed for 60 minutes in mice fed the CD or HC diets. After reperfusion, the left lobe of the liver was taken out via an abdominal incision, gently pressuring the abdomen to allow the edge of the lobe to emerge as described before [15]. 2'-7'-dichlorofluorescein (DCF, 200mM, incubated for $45 \mathrm{~min}$ ) and tetramethylrhodamine ethyl ester (TMRE, $5 \mathrm{mM}$ incubated for $30 \mathrm{~min}$ ) to monitor $\underline{\text { reactive oxygen species generation and mitochondrial membrane potential, respectively, }}$ were added on the lobe for 30-45 minutes and imaged using one photon excitation at $485 \mathrm{~nm}$ and emission at $380-520 \mathrm{~nm}$ for the DCF and excitation at $516 \mathrm{~nm}$ and emission at $582-677 \mathrm{~nm}$ for the TMRE. Both emissions were acquired with internal spectral detector (PMT) at high speed (31 frames, line average 4) in order to avoid movement from animal breathing and heart beating during acquisition. Images were collected in a 512 x 512 format, zoom 1.7 and pinhole completely open. Imaging of the liver of living mice was performed using spectral confocal inverted microscope Leica TCS SP5, equipped with $63 \mathrm{x}$ glycerol immersion (NA1.4) objective and near infrared laser $1.2 \mathrm{ps}$ (titanium-saphire IR MaiTai Broad laser, 710-990nm, Broad Band, Spectra Physics), resonant scan (video rate speed) and incubation heating system.

\section{Biochemical determinations.}


TG, FFA and cholesterol in plasma or tissue were measured by the Centro de Diagnóstico Medico (Hospital Clinic, Barcelona, Spain). In brief,....

\section{Total and unsterified cholesterol analysis.}

Total cholesterol determination in hepatic or mitochondrial extracts $(5 \mathrm{mg})$ was performed upon saponification (alcoholic $\mathrm{KOH}$ at $60^{\circ} \mathrm{C}$ for 30 minutes) followed by addition of $10 \mathrm{ml}$ of hexane and $3 \mathrm{ml}$ of distilled water. Hexane layer aliquots were evaporated under nitrogen and used for cholesterol measurement. Free cholesterol was determined from unsaponified samples and analyzed by HPLC using Bondapak C18 10$1 \mathrm{~m}$ reversed-phase column (30 cm, $4 \mathrm{~mm}$ inner diameter; Waters, Barcelona, Spain), and 2-propanol:acetonitrile:water (60:30:10) as the mobile phase at a flow rate of $1 \mathrm{ml} / \mathrm{min}$ as described [10].

\section{Total and mitochondrial GSH determination.}

GSH and GSSG in hepatic homogenates or mitochondrial fraction were measured after $10 \%$ trichloroacetic acid precipitation by HPLC as described $[16,17]$. In some cases, total GSH levels in either cytosol or mitochondria were determined by the recycling assay as reported previously $[18,19]$.

\section{Liver damage and lipid peroxidation.}

After reperfusion, livers were fixed with.... and sections $(5 \mu \mathrm{m})$ stained with hematoxylin and eosin (H\&E) using standard methods and slides were examined with a Zeiss Axioplan microscope (Göttingen, Germany) equipped with a Nikon DXM1200F digital camera (Tokyo, Japan). Serum ALT levels were measured by the Centro de Diagnóstico Medico (Hospital Clinic, Barcelona, Spain). Lipid peroxidation in liver samples was determined after the production of malondialdehyde (MDA) using the thiobarbituric acid (TBA) method as described previously [13]. 


\section{StAR expression}

Hepatic StAR mRNA levels from ob/ob mice were determined by quantitative real-time RT-PCR analyses (primer sense, CCACCCCTAGCACGTGGA; primer antisense, TCCTGGTCACTGTAGAGAGTCTCTTC) using the.....(equipment description). RNA was extracted by TRIzol (Invitrogen) method following the manufacturer's instructions under sterile conditions. RNA integrity and concentration were analysed by NanoDrop Spectrophotometer. Real-time PCR was performed with the iScript One-Step RT-PCR kit SYBR Green (Bio-Rad) with the following profile: $50 \mathrm{ng}$ of total RNA, $600 \mathrm{nM}$ of primers and $12.5 \mu \mathrm{l}$ of $2 \mathrm{X}$ Reaction Mix in a total volume of $25 \mu \mathrm{l}$ were incubated 10 $\min$ at $50^{\circ} \mathrm{C}, 5 \mathrm{~min}$ at $95^{\circ} \mathrm{C}$, followed for 45 cycles at $95^{\circ} \mathrm{C}$ for $10 \mathrm{~s}, 59^{\circ} \mathrm{C}$ for $30 \mathrm{~s}$ and $72^{\circ} \mathrm{C}$ for $30 \mathrm{~s}$. Each reaction was done in triplicate and the threshold values $(\mathrm{C} \tau)$ for each mRNA were substracted from that of HRP mRNA, as a housekeeping gene, averaged and converted from log-linear to linear term.

\section{Statistical Analyses}

Results are expressed as mean SD. Experiments were performed routinely with at least 4 to 6 animals per group. Statistical comparison of the mean values was performed using the Student's t-test for unpaired data. MORE DESCRIPTION 


\section{RESULTS}

\section{Nutritional and genetic models of hepatic steatosis with distinct lipid profile.}

Since our aim was to address the specific role of individual lipids accumulating in hepatic steatosis to I/R injury, we used nutritional models of fatty livers exhibiting a different lipid profile. Choline is an essential nutrient required for efficient phosphatidylcholine synthesis necessary for the packaging and export of VLDL particles to extrahepatic organs. Feeding mice a CD diet results in hepatic steatosis with predominant TG content and unchanged cholesterol levels compared to chow-fed mice (Fig 1A), consistent with previous reports [10, 20, 21]. In contrast, increased hepatic cholesterol levels but unchanged TG content are observed in mice fed the HC diet (Fig 1B). Filipin staining and HPLC analyses indicated that most of the total cholesterol levels are unsterified (Fig. 1B, D). In line with our prior observations, the content of FFA increased with respect to chow fed mice to a similar level in both CD or HC fed mice (Fig 1C). Oil red staining indicated the appearance of microvesicular steatosis with a strong filipin staining in $\mathrm{HC}$-fed mice, while the steatosis of CD-fed mice was macrovesicular compared to HC-fed mice with little filipin staining (Fig 1D, E). Unlike CD or HC-fed mice, the liver of ob/ob mice is characterized by the presence of TG, FFA and cholesterol as revealed by biochemical determinations (Supplemental Fig 1), while oil-red and filipin staining indicated the appearance of large lipid droplets indicative of macrovesicular steatosis and free cholesterol accumulation (Fig. 1D, E). Thus, these nutritional and genetic models induce fatty liver with different lipid composition mainly enriched in TG or cholesterol and similar FFA content.

HC-fed mice and ob/ob mice are more sensitive to hepatic I/R injury than CD-fed mice. 
We next examined the susceptibility of $\mathrm{CD}$ or $\mathrm{HC}$ fed mice to partial hepatic I/R injury. Compared to sham controls, normothermic ischemia (90 minutes) followed by 6 hours after reperfusion resulted in significant release of serum ALT in control chow-fed mice and CD-fed mice (Fig. 2A). However, the magnitude of ALT release in HC fed mice was significantly greater compared to control or CD-fed mice (Fig. 2A). In line with this outcome, histology analyses indicated that the liver damage in HC fed mice was more severe compared to mice fed the CD diet (Fig 2B). In addition, ob/ob mice, which exhibit genetic macrovesicular steatosis, were markedly sensitive to hepatic $\mathrm{I} / \mathrm{R}$ injury compared to CD-fed mice, determined by transaminase release (Fig 2C) or histology analyses (Fig 2D), similar to the damage seen in mice fed the HC diet. Consistent with previous findings, the susceptibility of $\mathrm{ob} / \mathrm{ob}$ mice to hepatic I/R was greater than that of ED fed mice [3]. Thus, although the morphological and qualitative appearance of hepatic steatosis of $\mathrm{HC}$-fed and ob/ob mice is strikingly different, displaying a microvesicular and macrovesicular pattern, respectively, both exhibit a high sensitivity towards hepatic I/R injury. Despite that TG are a predominant determinant of hepatic steatosis and a major component of lipid droplets [22], these data suggest that the presence of cholesterol rather than TG plays a significant role in the susceptibility to hepatic I/R injury.

Mitochondrial cholesterol potentiates mitochondrial GSH depletion following I/R.

Cholesterol is a critical bilayer component that distributes heterogenously among cell membranes. Although mitochondria are cholesterol-poor organelles, mitochondrial cholesterol has emerged as an important player in pathophysiology, including liver diseases such as steatohepatitis [11]. Therefore, we next evaluated the status of mitochondrial cholesterol in mice fed $\mathrm{HC}$ or CD diets. As seen, HC-fed mice but not CD-fed mice exhibited increased mitochondrial cholesterol content, and this particular 
cholesterol pool was insensitive to I/R (Fig 3A), paralleling the increase seen in hepatic extracts (Fig. 1B). Moreover, since GSH homeostasis plays a critical role in hepatic I/R injury, we examined the status of total GSH vs that of the specific pool of GSH in mitochondria during I/R. The level of GSH in hepatic extracts decreased following I/R regardless of the nutritional regimen and independent of the hepatic lipid profile (Fig. 3B). In contrast, the hepatic mitochondrial GSH content decreased significantly in sham HC-fed mice (Fig 3C), consistent with the negative impact of mitochondrial cholesterol in mitochondrial GSH transport $[18,23]$. I/R depleted mitochondrial GSH in control and CD-fed mice, likely reflecting the decrease of cytosolic GSH (Fig. 3B). However, this effect of $\mathrm{I} / \mathrm{R}$ on mitochondrial GSH depletion was further potentiated in HC-fed mice (Fig. 3C) because of the additional contribution of mitochondrial cholesterol loading. Thus, these findings correlate the susceptibility of HC-fed mice with increased mitochondrial cholesterol trafficking and subsequent mitochondrial GSH depletion.

In vivo confocal imaging of fatty livers from mice fed CD or HC diets following I/R.

The mechanisms underlying hepatic $I / R$ injury are multifactorial, with the overgeneration of reactive oxygen species (ROS) playing a critical role. Moreover, mitochondria not only are a major source of ROS but they are instrumental in the predominant necrotic cell death during $\mathrm{I} / \mathrm{R}$ injury via mitochondrial permeability transition (MPT) [24, 25]. Having observed the depletion of mitochondrial GSH in fatty livers of HC-fed mice, which are particularly susceptible to I/R damage, we next analyzed the burst of ROS and mitochondrial membrane potential in vivo by twophoton confocal imaging of livers during I/R. ROS stimulation and mitochondrial membrane potential were recorded by DCF and TMRM labeling and imaged with an internal spectral detector at high speed to avoid the movement from animal breathing 
and heart beating during acquisition. As seen, while I/R stimulated the ROS generation and mitochondrial depolarization in control livers, these effects were markedly enhanced in the ischemic livers of HC-fed mice but not in mice fed the CD diet (Fig. 4), consistent with the preceding findings on the mitochondrial GSH status, which is known to regulate MPT. Thus, HC feeding potentiates ROS stimulation and mitochondrial depolarization in vivo during hepatic I/R.

\section{Atorvastatin and squalene synthase inhibition prevent mitochondrial cholesterol loading and protect ob/ob mice from I/R injury.}

Given the above findings pointing to cholesterol as a potential culprit in the sensitization to hepatic $\mathrm{I} / \mathrm{R}$ injury, we next examined the effect of cholesterol downregulation in ob/ob mice. We targeted two steps in the mevalonate pathway of the de novo cholesterol synthesis, namely, HMGCoA R, and the squalene synthase (Fig 5A), examining the impact on the mitochondrial cholesterol trafficking. Although there was a trend for lower cholesterol levels in hepatic extracts of ob/ob mice after atorvastatin (Fig 5B), it significantly attenuated the increase in mitochondrial cholesterol (Fig. 5C). Biochemical analyses indicated that the atorvastatin was effective in decreasing the cholesterol levels in plasma (Fig 5D), suggesting that enhanced LDL uptake may contribute to the maintenance of total hepatic cholesterol pool following statin therapy and that mitochondrial cholesterol derives predominantly from de novo synthesis. Moreover, consistent with the increased mitochondrial cholesterol, ob/ob mice exhibited enhanced expression of StAR (Fig 5E), a mitochondrial cholesterol transporting polypeptide [26, 27]. Interestingly, atorvastatin therapy blunted StAR expression in ob/ob mice (Fig 5E).

Since the inhibition of HMGCoAR can result in nonspecific effects independent of eholesterel regulation, We next tested the effect of squalene synthase inhibition by YM- 
53601, which is expected to prevent cholesterol synthesis from squalene without interference in the formation of non-sterol branch of the mevalonate pathway (Fig 5A). Neither atorvastatin nor the squalene synthase inhibitor affected the total cholesterol (14.3-16.6 $\mu \mathrm{g}$ cholesterol/mg protein) or the mitochondrial cholesterol pool $(6.5-7.8 \mu \mathrm{g}$ cholesterol/mg protein) in lean mice. However, the effect of YM-53601 on total cholesterol levels was similar to that observed by atorvastatin, but was able to significantly ameliorate the increase of the hepatic mitochondrial cholesterol in ob/ob mice (Fig 5B, C). In paralell with these observations, both atorvastatin and YM-53601 protected $\mathrm{ob} / \mathrm{ob}$ mice against $\mathrm{I} / \mathrm{R}$ injury based on serum ALT values and histology examination following I/R injury (Fig. 6). Moreover, the H\&E staining suggested that both therapeutic options ameliorated the hepatic steatosis of ob/ob mice that reflected decreased TG deposition (Supplemental Figure 2). Thus, these findings suggest that the newly synthesized cholesterol traffics to mitochondria and that its downregulation by statins of squalene synthase inhibition protects ob/ob mice from I/R liver injury.

\section{Restoration of mitochondrial GSH in ob/ob mice by atorvastatin and squalene} synthase inhibition following I/R.

Mitochondrial GSH is a critical antioxidant that regulates hepatocyte survival in response to oxidative stress, and hepatic I/R injury [28]. Since mitochondrial GSH regulation is dependent on cholesterol-mediated control of mitochondrial membrane dynamics, we next examined the effect of atorvastatin and YM-53601 on mitochondrial GSH homeostasis in ob/ob mice following I/R. Consistent with the effects observed on mitochondrial cholesterol pool, atorvastatin and YM-53601 restored the hepatic mitochondrial GSH content of sham control ob/ob mice to levels close to those observed in lean mice (Fig 7). Importantly, while this particular pool of GSH decreased further in ob/ob mice during $\mathrm{I} / \mathrm{R}$, both therapies significantly increased the 
mitochondrial GSH levels in ob/ob mice following I/R (Fig 7), in agreement with the effect of atorvastatin and YM-53601 on the mitochondrial cholesterol content (Fig 5C). Similar protective findings were observed when the pool of mGSH is restored by GSH ethyl ester (not shown), a permeable form of GSH [21]. Consistent with the reversal of the mitochondrial GSH stores, atorvastatin and the squalene synthase inhibitor attenuated oxidative stress determined by the increase in MDA levels (Supplemental Fig 3). Thus, these findings indicate that the regulation of mitochondrial cholesterol content modulate the levels of mitochondrial GSH whose replenishment attenuates hepatic I/R injury. 


\section{DISCUSSION}

The mechanisms whereby hepatic steatosis sensitizes to I/R injury remain incompletely understood, and their elucidation may provide ways to increase the graft load available for use in diverse clinical settings requiring $\mathrm{I} / \mathrm{R}$. In this study, we analyzed the susceptibility of fatty livers with specific lipid profiles to I/R damage. Using nutritional and genetic models of hepatic steatosis, we observed that regardless of the qualitative appearance of steatosis (micro vs macrosteatosis), the presence of cholesterol, particularly in mitochondria, plays a previously unrecognized role in normothermic ischemic injury. Previous studies indicated that livers with microsteatosis induced by $\mathrm{CD}$ diet feeding were less sensitive to $\mathrm{I} / \mathrm{R}$ damage than those with macrosteatosis seen in ob/ob mice [3]. Although we confirm this outcome here, we believe the relative qualitative aspect of steatosis plays a minor role in hepatic I/R susceptibility. While the steatosis of CD-fed mice may be microvesicular with respect to that of ob/ob mice, it is more macrovesicular than that of HC-fed mice. Moreover, although HC-fed mice exhibit microvesicular steatosis, these livers are as sensitive to I/R-induced damage as livers of ob/ob mice with macrovesicular steatosis. Rather than the relative contribution of micro vs macrosteatosis in discriminating the susceptibility to I/R injury, we provide evidence that hepatic cholesterol plays a so far unappreciated role in the sensitization of fatty liver to ischemic injury. CD-fed mice is characterized by steatosis with TG and FFA accumulation but unchanged cholesterol, in contrast to livers from HC-fed mice which exhibit a predominant cholesterol loading. Our findings showing a critical role of cholesterol in this process is consistent with previous reports in $\mathrm{LDLr}^{-/-}$mice or rabbits fed a high-cholesterol diet $[29,30]$. Moreover, the susceptibility of $\mathrm{LDLr}^{-/-}$mice to hepatic I/R injury was ameliorated by anti TNF antibodies [29], pointing to a critical role of TNF in hepatic I/R damage. Considering previous findings favoring the 
transition of steatosis to steatohepatitis by cholesterol [10], the present findings broaden the implications of cholesterol in liver pathology.

The role of cholesterol in sensitizing to normothermic hepatic $I / R$ injury is likely mediated by its trafficking and accumulation in mitochondria and subsequent depletion of mitochondrial GSH. Indeed, we observed in vivo mitochondrial depolarization and ROS generation by confocal imaging in $\mathrm{HC}$-fed mice as opposed to $\mathrm{CD}$-fed mice, consistent with the critical role of mitochondrial GSH in cell survival and defense against oxidative stress. Mitochondrial cholesterol loading is known to perturb membrane dynamics affecting selective carriers, including the GSH mitochondrial transporter but not the S-adenosyl-L-methionine (SAM) carrier [31]. Hence, unlike CDfed mice substantial hepatic mitochondrial GSH depletion is observed in HC-fed and ob/ob mice, both exhibiting marked susceptibility to I/R. Although I/R itself depletes hepatic GSH levels and mitochondrial GSH in paralell, the decrease of this latter specific pool is more dramatic upon mitochondrial cholesterol loading as seen in HCfed and ob/ob mice. Quite interestingly, we observed for the first time that livers from ob/ob mice exhibit enhanced expression of StAR, a mitochondrial cholesterol transporting polypeptide [26, 27], consistent with the mitochondrial cholesterol accumulation observed in these samples. Although we did not address the contribution of StAR in the regulation of mitochondrial cholesterol in ob/ob mice, it is conceivable that it may play a significant role as observed in human hepatocytes. Overexpression of StAR in HepG2 cells has been reported to cause the upregulation of 27hydroxycholesterol, a read-out metabolite of increased mitochondrial cholesterol pool [32]. Moreover, StAR silencing by siRNA has been shown to decrease this pool of cholesterol sensitizing human hepatocarcinoma cells to chemotherapy [33]. 
Additional evidence to support a role for cholesterol, particularly in mitochondria, in the susceptibility of ob/ob mice to hepatic I/R damage is provided by preventing cholesterol synthesis in the mevalonate pathway. Atorvastatin which targets HMGCoA R, the regulatory enzyme in the de novo cholesterol synthesis from acetyl-CoA, downregulates mitochondrial cholesterol loading, and consequently prevents mitochondrial GSH depletion during I/R. Quite intriguingly, atorvastatin also prevented the upregulation of StAR by an unknown mechanism, consistent with the above described effects on mitochondrial cholesterol. By targeting HMGCoA R, statins may have broad effects far from just preventing cholesterol synthesis, as the conversion of mevalonate into farnesyl-PP can branch into non-sterol intermediates including isoprenoids, which can regulate a number of target proteins posttranslationally. To confirm if the therapeutic effect of atorvastatin in ob/ob mice against hepatic I/R injury is due to the downregulation of cholesterol and its trafficking to mitochondria, we inhibited squalene synthase, which catalyzes the first committed step in cholesterol synthesis. Squalene synthase inhibition paralleled the effects observed with atorvastatin with respect to mitochondrial cholesterol depletion and subsequent mitochondrial GSH restoration, strongly suggesting that the beneficial effects of atorvastatin in hepatic I/R susceptibility is due to cholesterol regulation. Consistent with these effects, previous studies have shown that simvastatin reduces the mortality and hepatic injury after hemorrhage/resuscitation in rats or during liver I/R injury $[34,35]$. In comparing the effect of atorvastatin in StAR and mitochondrial cholesterol regulation in ob/ob mice, it appears that the statin prevented StAR upregulation while it attenuated mitochondrial cholesterol loading, which suggests the possibility for the involvement of other StAR family members. Whether or not MLN64, which has been shown to mediate the egress of cholesterol from endosomes to mitochondria in the absence of Niemann-Pick type C1 
protein [36], is involved in the upregulation of mitochondrial cholesterol in ob/ob mice remains to be established. Finally, it may give the impression that the pharmacological agents we use to modulate cholesterol levels exhibited unspecific effects reflected in the downregulation of $\mathrm{TG}$ levels in ob/ob mice. However, while these agents target HMGCoA reductase and squalene synthase specifically to prevent cholesterol synthesis, they may cause secondary effects in the regulation of other lipids including TG. Ob/ob mice is known to exhibit endoplasmic reticulum (ER) stress [36] and cholesterol upregulation has been shown to contribute to this response [37], which in turn leads to activation of ER-based transcription factors stch as SREBPs [8,9]. Thus, it is conceivable that cholesterol downregulation in ob/ob mice (by atorvastatin or YM53601) may secondarily contribute to SREBP1c downregulation via decreased ER stress, accounting for the reduction in TG content.

In summary, by comparing side-by-side the susceptibility of fatty livers with disparate lipid composition, we uncovered a critical role of mitochondrial cholesterol in the sensitization of fatty livers againt ischemic injury and that this effect is due to mitochondrial GSH depletion. Consistent with this outcome, previous studies reported the depletion of this pool of GSH during hepatic I/R damage, and its restoration by GSH ethyl ester [37] or SAM [38] protected steatotic livers against I/R damage. Although this concept remains to be tested in human fatty liver, mitochondrial GSH depletion has been shown in nonalcoholic steatohepatitis [39] which is associated with increased free cholesterol accumulation and StAR overexpression [40]. If confirmed the role of cholesterol and its accumulation in mitochondria in human fatty livers and its correlation with graft failure, then analyzing the presence of cholesterol or cholesterolrelated proteins such as StAR in steatotic donor grafts may represent a valuable discriminating tool to expand the pool of available organs for transplantation. 


\section{ACKNOWLEDGMENTS}

The work was supported by grants SAF2006-06780, SAF2008-02199, SAF2008-04974 and SAF2009-11417 (Plan Nacional de I+D), PI070193 and PI09/00056 (Instituto de Salud Carlos III), by CIBEREHD from the Instituto Carlos III, the Fundación Mutua Madrileña and the center grant P50-AA-11999 Research Center for Liver and Pancreatic Diseases, US National Institute on Alcohol Abuse and Alcoholism, USA. 


\section{REFERENCES}

[1]. Selzner M, Clavien PA. Fatty liver in liver transplantation and surgery. Sem Liver Dis 2001; 21: 105-113.

[2]. Hakamada K, Sasaki M, Takahashi K, Umehara Y, Konn M. Sinusoidal flow block after warm ischemia in rats with diet induced fatty liver. J Surg Res 1997; 70: 1220.

[3]. Selzner N, Selzner M, Jochum W, Amann-Vesti B, Graf R, Clavien PA. Mouse liver with macrosteatosis are more susceptible to normothermic ischemic injury than those with microsteatosis. J Hepatol 2006; 44:694-701.

[4]. El-Brady AM, Moritz W, Contaldo C, Tian Y, Graf R, Clavien PA. Prevention of reperfusion injury and microcirculatory failure in macrosteatosis mouse liver by omega-3 fatty acids. Hepatology 2007; 45:855-863.

[5]. Yoong KF, Gunson BK, Neil DA, Mirza DF, Mayer AD, Buckels JA et al. Impact of donor liver microvesicular steatosis on the outcome of liver retransplantation. Transplant Proc 1999; 31: 550-551.

[6]. Fishbein TM, Fiel MI, Emre S, Cubukcu O, Guy SR, Schwartz ME et al. Use of livers with microvesicular fat safely expands the donor pool. Transplantation 1997; 64: 248-251.

[7]. Urena MA, Ruiz-Delgado FC, Gonzalez EM, Segurola CL, Romero CJ, Garcia IG et al. Assessing risk of the use of livers with macro and microsteatosis in a liver transplant program. Transplant Proc 1998; 30: 3288-3291.

[8]. Browning JD, Horton JD, Molecular mediators of hepatic steatosis and liver injury, J. Clin. Invest.2004; 114: 147-152. 
[9]. Brown MS, Goldstein JL, The SREBP pathway: regulation of cholesterol metabolism by proteolysis of a membrane-bound transcription factor, Cell 1997; 89: $331-340$.

[10]. Mari M, Caballero F, Colell A, Morales A, Caballeria J, Fernandez A et al. Mitochondrial free cholesterol loading sensitizes to TNF- and Fas-mediated steatohepatitis. Cell Metab 2006; 4: 185-198.

[11]. Garcia-Ruiz C, Mari M, Colell A, Morales A, Caballero F, Montero J, et al. Mitochondrial cholesterol in health and disease. Histol Histopathol 2009; 24: 117-132.

[12]. Llacuna L, Mari M, Garcia-Ruiz C, Fernandez-Checa JC, Morales A. Critical role of acidic sphingomyelinase in murine hepatic ischemia-reperfusion injury. Hepatology 2006; 44: 561-572.

[13]. Llacuna L, Marí M, Lluis JM, García-Ruiz C, Fernández-Checa JC, Morales A. Reactive oxygen species mediate liver injury through parenchymal nuclear factor-kB inactivation in prolonged ischemia/reperfusion. Am J Pathol. 2009;174:1776-1785.

[14]. Colell A, García-Ruiz C, Lluis JM, Coll O, Mari M, Fernández-Checa JC. Cholesterol impairs the adenine nucleotide translocator-mediated mitochondrial permeability transition through altered membrane fluidity. J Biol Chem 2003; 278:33928-33935.

[15]. Thiberge S, Blazquez S, Baldacci P, Renaud O, Shorte S, Menard R, Amino R. In vivo imaging of malaria parasites in the murine liver. Nat Protoc. $2007 ; 2: 1811-1818$. [16]. Fariss MW, Reed DJ. High-performance liquid chromatograpy of thiols and disulfides: dinitrophenol derivatives. Methods Enzymol. 1987;143:101-109.

[17]. García-Ruiz C, Morales A, Ballesta A, Rodés J, Kaplowitz N, Fernández-Checa JC. Effect of chronic ethanol feeding on glutathione and functional integrity of 
mitochondria in periportal and perivenous rat hepatocytes. J Clin Invest. 1994;94:193201.

[18]. Colell A, Garcia-Ruiz C, Miranda M, Ardite E, Mari M, Morales A, et al. Selective glutathione depletion of mitochondria by ethanol sensitizes hepatocytes to tumor necrosis factor. Gastroenterology 1998; 115: 1541-1551.

[19]. Lluis J M, Colell A, Garcia-Ruiz C, Kaplowitz N, Fernandez-Checa J C. Acetaldehyde impairs mitochondrial glutathione transport in HepG2 cells through endoplasmic reticulum stress. Gastroenterology 2003; 124: 708-724.

[20]. Behari J, Yeh TH, Krauland L, Otruba W, Cieply B, Hauth B, Apte U, et al. Liver-specific beta-catenin knockout mice exhibit defective bile acid and cholesterol homeostasis and increased susceptibility to diet-induced steatohepatitis. Am J Pathol 2010; 176: 744-753.

[21]. Caballero F, Fernandez A, Matias N, Martinez L, Fucho R, Elena M et al. Specific contribution of methionine and choline in nutritional non-alcoholic steatohepatitis: Impact on mitochondrial S-adenosyl-L-methionine and GSH. J Biol Chem 2010; 18528-18536.

[22]. Farese RV Jr, Walther TC. Lipid droplets finally get a little R-E-S-P-E-C-T. Cell $2009 ; 139: 855-860$.

[23]. Colell A, García-Ruiz C, Morales A, Ballesta A, Ookhtens M, Rodés J,et al. Transport of reduced glutathione in hepatic mitochondria and mitoplasts from ethanoltreated rats: effect of membrane physical properties and S-adenosyl-L-methionine. Hepatology 1997; 26:699-708.

[24]. Jaeschke H, Lemasters JJ. Apoptosis versus oncotic necrosis in hepatic ischemia/reperfusion injury. Gastroenterology 2003; 125: 1246-1257. 
[25]. Kim JS, He L, Lemasters JJ. Mitochondrial permeability transition: a common pathway to necrosis and apoptosis. Biochem Biophys Res Commun. 2003;304:463-470. [26]. Soccio RE, Breslow JL. Intracellular cholesterol transport. Arterioscler Thromb Vasc Biol 2004; 24:1150-1160.

[27]. Maxfield FR, Tabas I. Role of cholesterol and lipid organization in disease. Nature 2005, 438:612-621.

[28]. Marí M, Morales A, Colell A, García-Ruiz C, Fernández-Checa JC. Mitochondrial glutathione, a key survival antioxidant. Antioxid Redox Signal 2009; 11: $2685-2700$.

[29]. Mori N, Horie Y, Yuji N, Wolf R, Granger N. Hepatic microvesicular responses to ischemia-reperfusion in low-density lipoprotein receptor knockout mice. Am J Physiol 2000; 279:G1257-G1264.

[30]. Fusai G, Glantzounis GK, Hafez T, Yang W, Quaglia A, Sheth H et al. Nacetylcysteine amelioreates the late phase of liver ischemia/reperfusion injury in the rabbit with hepatic steatosis. Clin Sci 2005; 109:465-473.

[31]. Fernández A, Colell A, Caballero F, Matías N, García-Ruiz C, Fernández-Checa JC. Mitochondrial S-adenosyl-L-methionine transport is insensitive to alcohol-mediated changes in membrane dynamics. Alcohol Clin Exp Res 2009; 33:1169-1180.

[32]. Hall EA, Ren S, Hylemon PB, Rodriguez-Agudo D, Redford K, Marques D, et al. Detection of the steroidogenic acute regulatory protein, StAR, in human liver cells. Biochim Biophys Acta 2005; 1733:111-119.

[33]. Montero J, Morales A, Llacuna L, Lluis JM, Terrones O, Basañez G, et al. Mitochondrial cholesterol contributes to chemotherapy resistance in hepatocellular carcinoma. Cancer Res 2008; 68: 5246-5256. 
[34]. Relja B, Lehnert M, Seyboth K, Bormann F, Hohn C, Czerny C, et al. Simvastatin reduces mortality and hepatic injury after hemorrhage/resuscitation in rats. Shock 2010; 34:46-54.

[36]. Charman M, Kennedy BE, Osborne N, Karten B. MLN64 mediates the egress of cholesterol from endosomes to mitochondria in the absence of functional Niemann-Pick type C1 protein. J Lipid Res 2010; 51:1023-1034.

[36]. Ozean U, Yilmaz E, Ozcan L, Furuhashi M, Vaillancourt E, Smith RO, et al. Chemical chaperones reduce ER stress and restore glucose homeostasis in a mouse model of type 2 diabetes. Science 2006; 313: 1137-1140.

[37]. Feng B, Yao PM, Li Y, Devlin CM, Zhang D, Harding HP et al. The endoplasmic reticulum is the site of cholesterol-induced cytotoxicity. Nat Cell Biol $2003 ; 5: 781-792$.

[37]. Grattagliano I, Vendemiale G, Lauterburg BH. Reperfusion injury of the liver: role of mitochondria and protection by glutathione ester. J Surg Res 1999; 86: 2-8.

[38]. Kaneshiro Y, Nakano H, Kumada K, Boudjema K, Kitamura N, Shimura H et al. Augmentation of mitochondrial reduced glutathione by S-adenosyl-L-methionine administration in ischemia-reperfusion injury of the rat steatotic liver induced by choline-methionine-deficient diet. Eur Surg Res 1998; 30: 34-42.

[39]. Serviddio G, Bellanti F, Tamborra R, Rollo T, Capitanio N, Roman AD et al. Uncoupling protein-2 (UCP2) induces mitochondrial proton leak and increases susceptibility of non-alcoholic steatohepatitis (NASH) liver to ischaemiareperfusion injury. Gut 2008; 57: 957-965. 
[40]. Caballero F, Fernández A, De Lacy AM, Fernández-Checa JC, Caballería J, García-Ruiz C. Enhanced free cholesterol, SREBP-2 and StAR expression in human NASH. J Hepatol 2009; 50: 789-796. 


\section{FIGURE LEGENDS.}

Figure 1. Nutritional and genetic models of micro and macrosteatosis. Livers from mice were fed a $\mathrm{HC}$ or $\mathrm{CD}$ diet or from ob/ob mice were analyzed for TG (A), cholesterol (B) or FFA (C) levels biochemically. In addition, samples were taken for oil red (D) or filipin (E) staining to monitor the appearance of neutral lipids or free cholesterol, respectively. Results are the mean $\mathrm{SD}$ of 4-6 mice per group. Images from D and E are representative of 4-6 mice per group. ${ }^{*} p<0.05$ vs control group. Magnification of images in D is $\mathrm{x} 20$, while in $\mathrm{E}$ is $\mathrm{x} 10$.

Figure 2. Differential susceptibility of steatotic livers to I/R injury. Mice fed CD or HC diets were analyzed for serum transaminase levels (A), or histology (B) to assess their sensitivity to I/R. Alternatively, ob/ob mice were used to determine transaminase values (C) or histology after I/R. Results are the mean SD of 4-6 mice per group. ${ }^{*} \mathrm{p}<0.05$ vs corresponding control groups with or without I/R. Magnification of images in B is x 20 .

Figure 3. Cholesterol and GSH compartmentation after I/R. Livers from mice fed CD or $\mathrm{HC}$ diets were processed for mitochondrial isolation to analyze cholesterol content (A). Alternatively, samples were used for GSH determination in homogenate (B) or mitochondria (C). Results are the mean SD of 5-7 mice. ${ }^{*} \mathrm{p}<0.05$ vs control mice. $\# \mathrm{p}<0.05$ vs control or CD-fed mice.

Figure 4. In vivo confocal imaging of mitochondrial polarization and ROS generation after I/R. In vivo two-photon confocal microscopy during partial I/R in mice fed CD or HC diets. ROS generation and mitochondrial polarization were assessed from DCF and TMRE fluorescence. The imaging of the liver of living mice was performed using spectral confocal inverted microscope Leica TCS SP5. The emissions were acquired with internal spectral detector (PMT) at high speed in order to avoid the movement from animal breathing and heart beating during acquisition. 
Figure 5. Effect of atorvastatin and squalene synthase inhibition on mitochondrial cholesterol trafficking. A, mevalonate pathway showing the branching of farnesyl-PP into cholesterol synthesis via squalene formation or the formation of non-sterol intermediates and the target of statins and YM-53601. Livers from lean and ob/ob mice were processed for cholesterol analyses in homogenates (B), mitochondria (C) as well as in plasma (D). E, shows the mRNA expression for StAR in livers from lean and ob/ob mice with or without atorvastatin treatment. Results are the mean SD of 4-6 mice per group. ${ }^{*} \mathrm{p}<0.05$ vs. control lean mice; $\# \mathrm{p}<0.05$ vs. ob/ob mice.

Figure 6. Atorvatatin and YM-53601 protect ob/ob mice from I/R injury. Ob/ob mice were pretreated with atorvastatin or YM-53601 for seven days before I/R at the dose of $10 \mathrm{mg} / \mathrm{kg}$ or $15 \mathrm{mg} / \mathrm{kg}$, respectively, or vehicle. 6 hours after reperfusion serum or liver biopsies were processed for assessment of transaminases (A) or histology (B). Results are the mean SD of 4-6 mice. ${ }^{*} \mathrm{p}<0.05 \mathrm{vs}$ ob/ob sham mice; $\# \mathrm{p}<0.05$ vs. vehicle-treated ob/ob mice. Magnification of images in B is x20.

Figure 7. Mitochondrial GSH levels in ob/ob mice following $\mathrm{I} / \mathrm{R}$ with or without atorvastatin or YM-53601 therapy. Samples from ob/ob mice following I/R with or without atorvastatin and YM-53601 therapy were processed for mitochondrial isolation and GSH determination. Results are the mean SD of 5-7 mice per group. ${ }^{*} \mathrm{p}<0.05$ vs lean or ob/ob sham mice, ${ }^{*}$ p $<0.05$ vs. ob/ob sham mice; $\# p<0.05$ vs. ob/ob mice following $\mathrm{I} / \mathrm{R}$. 


\section{SUPPLEMENTAL FIGURES}

Figure 1. Hepatic lipid profile in ob/ob mice. Livers from ob/ob mice were processed for biochemical determination of triglycerides, cholesterol and free fatty acids. Results are the mean $\mathrm{SD}$ of 5-7 mice per group. ${ }^{*} \mathrm{p}<0.05$ vs. lean mice.

Figure 2. Hepatic triglycerides in ob/ob mice following atorvastatin or YM53601 therapy. Liver samples from ob/ob mice with or without atorvastatin or YM-53601 were processed for biochemical determination of triglycerides. Results are the mean SD of 46 mice per group. ${ }^{*} \mathrm{p}<0.05$ vs. ob/ob mice with treatment.

Figure 3. Malondialdehyde levels in ob/ob mice following hepatic I/R. ob/ob mice were treated or not with atorvastatin or YM-53601 before undergoing hepatic I/R. Liver samples were collected after reperfusion $(6 \mathrm{hrs})$ and processed for MDA determination by the thibarbituric acid assay. Results are the mean SD of 4-6 mice per group. $p<0.05$ vs. sham ob/ob mice; $\#$ p $<0.05$ vs ob/ob mice. 
Figure 1

A

TRIGLYCERIDES

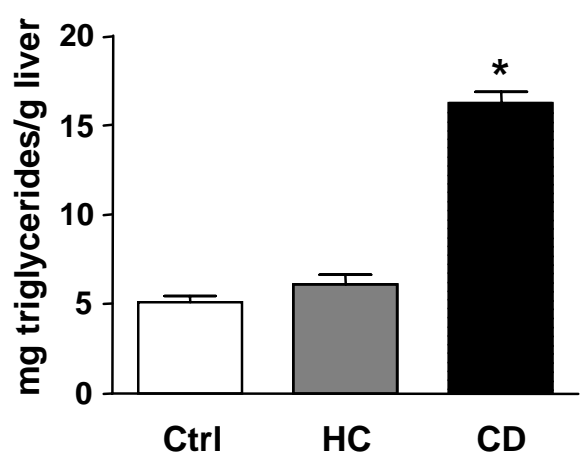

D

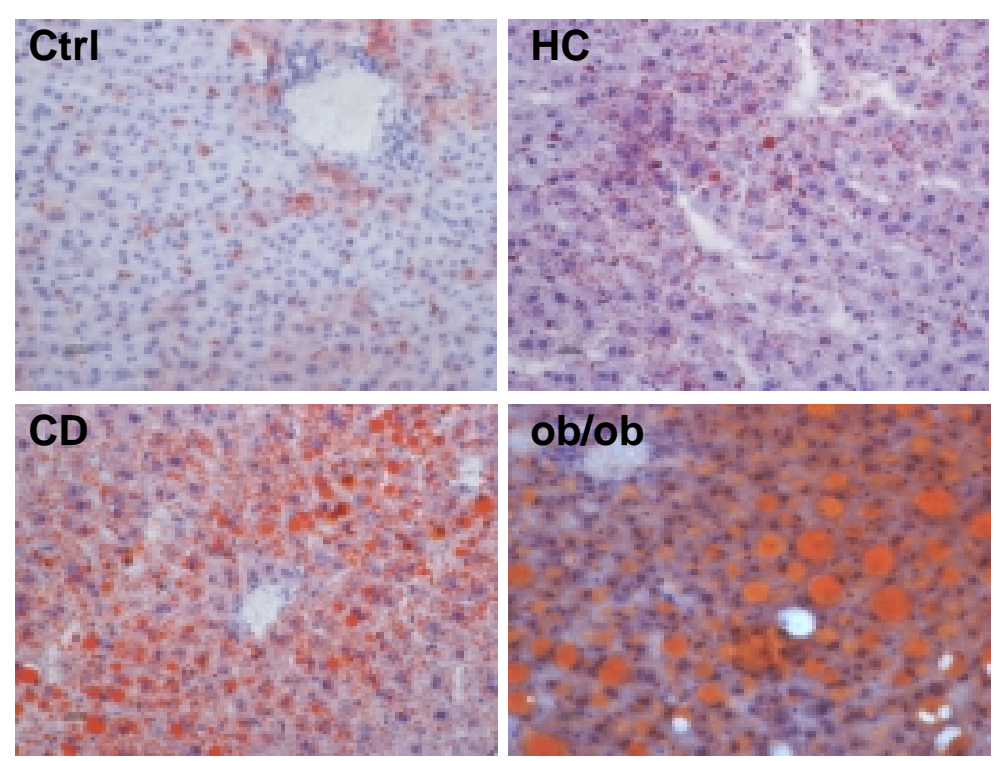

C

CHOLESTEROL

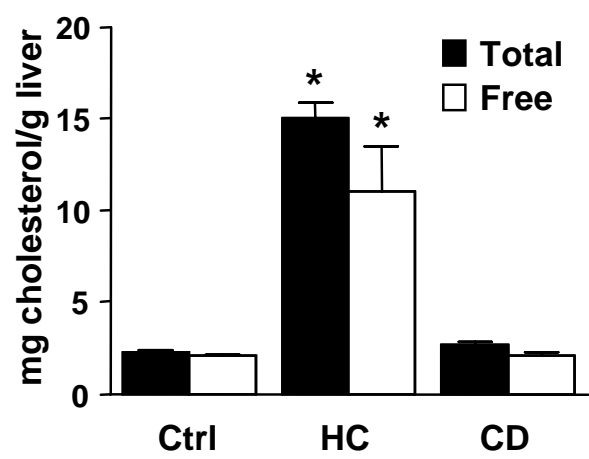

E
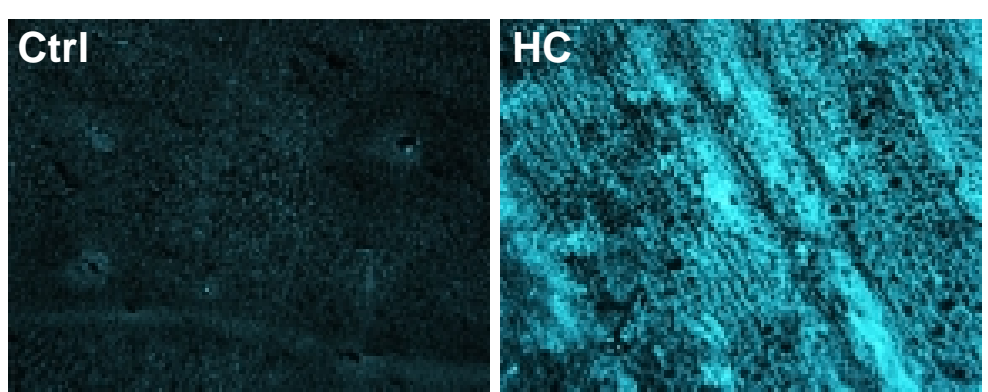

CD
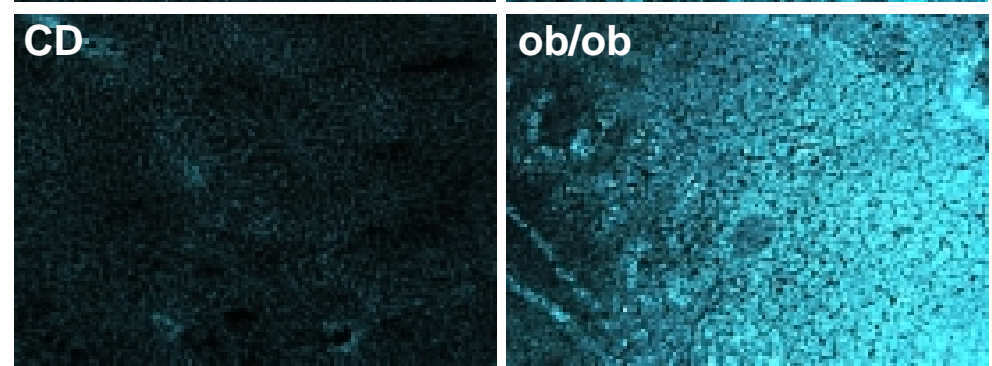
Figure 2

A

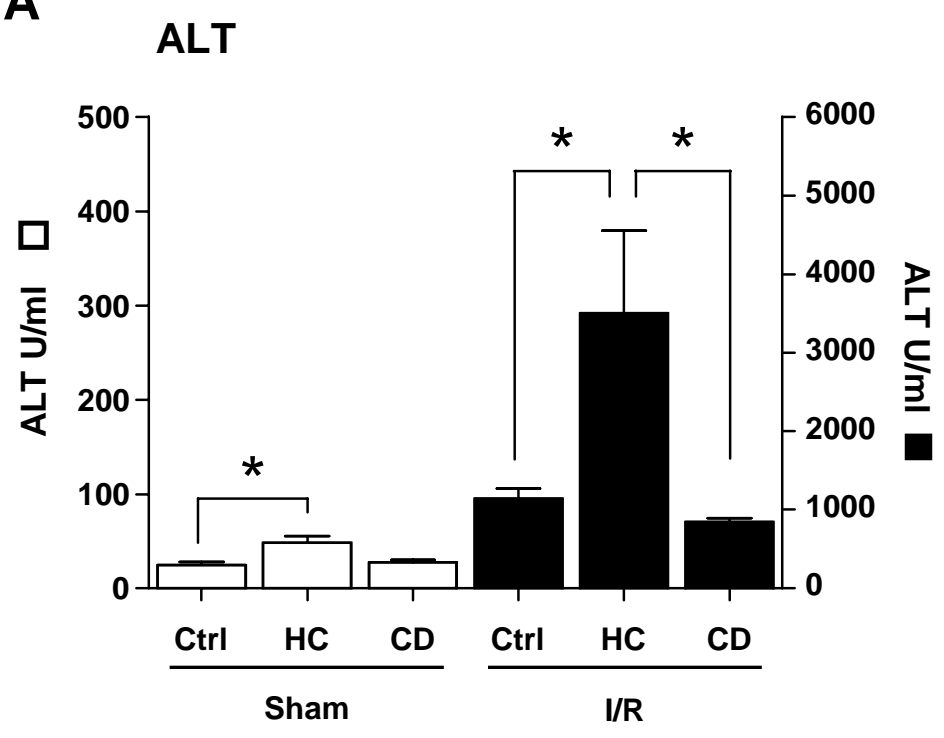

C

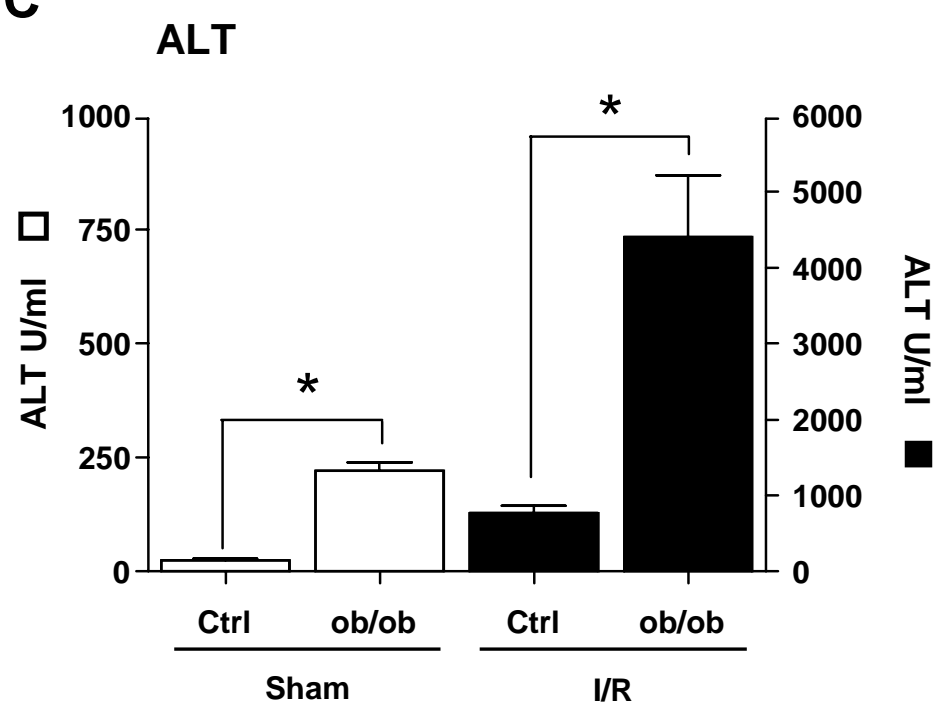

B

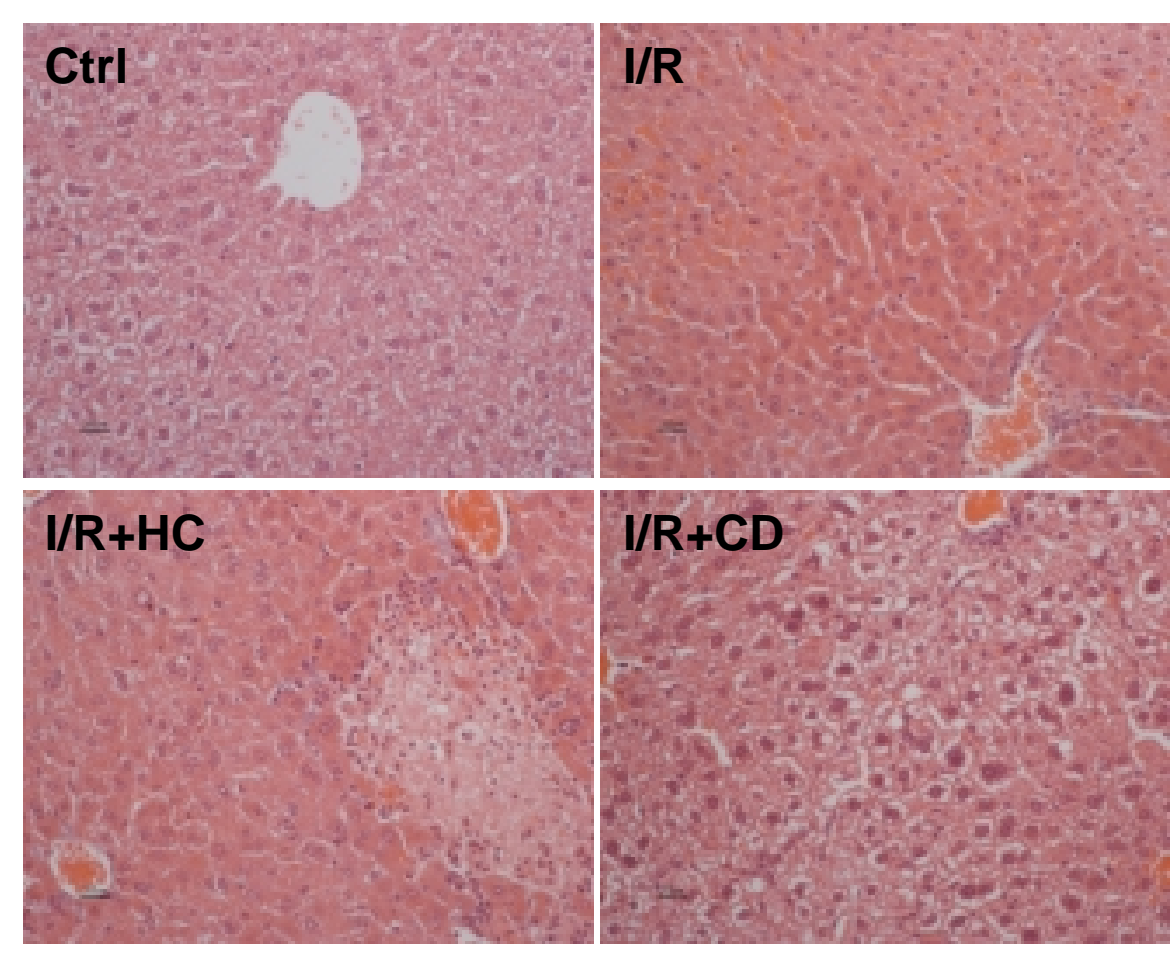

D

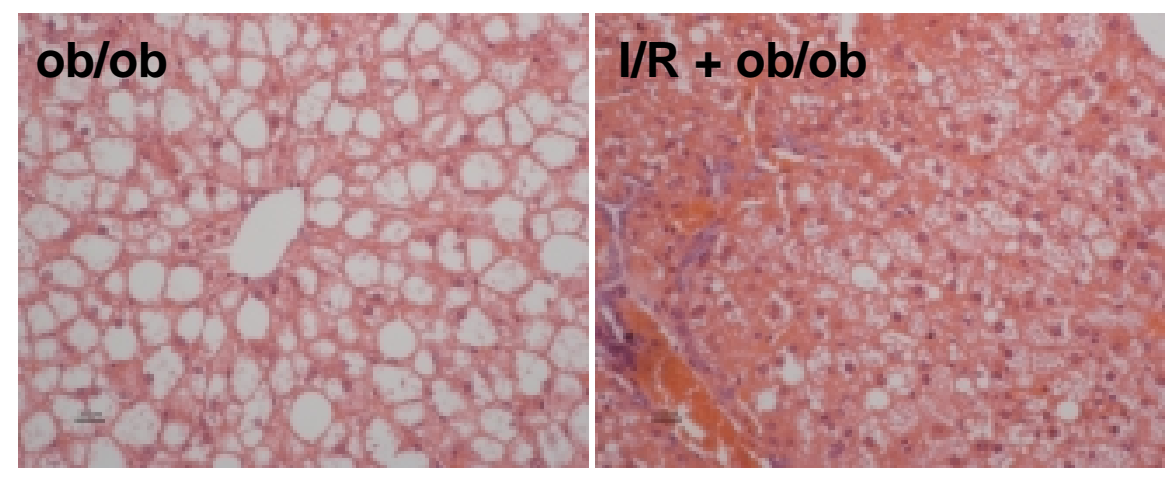


A

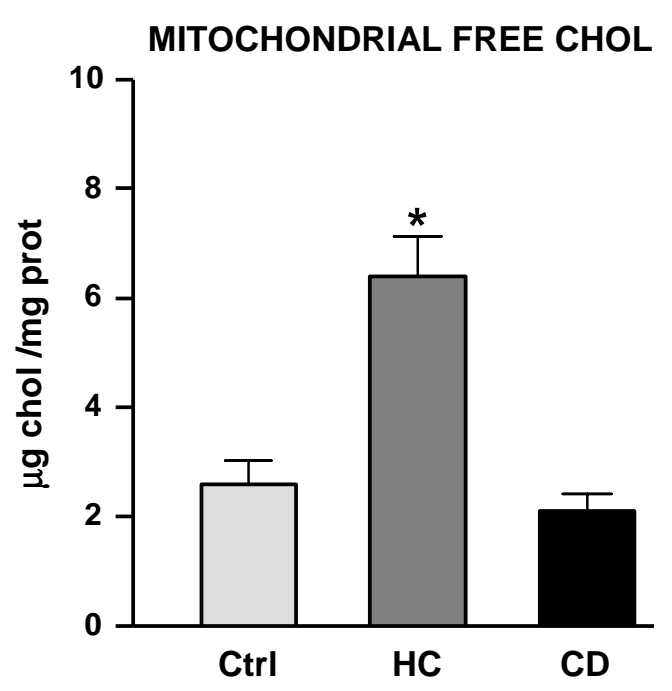

Figure 3
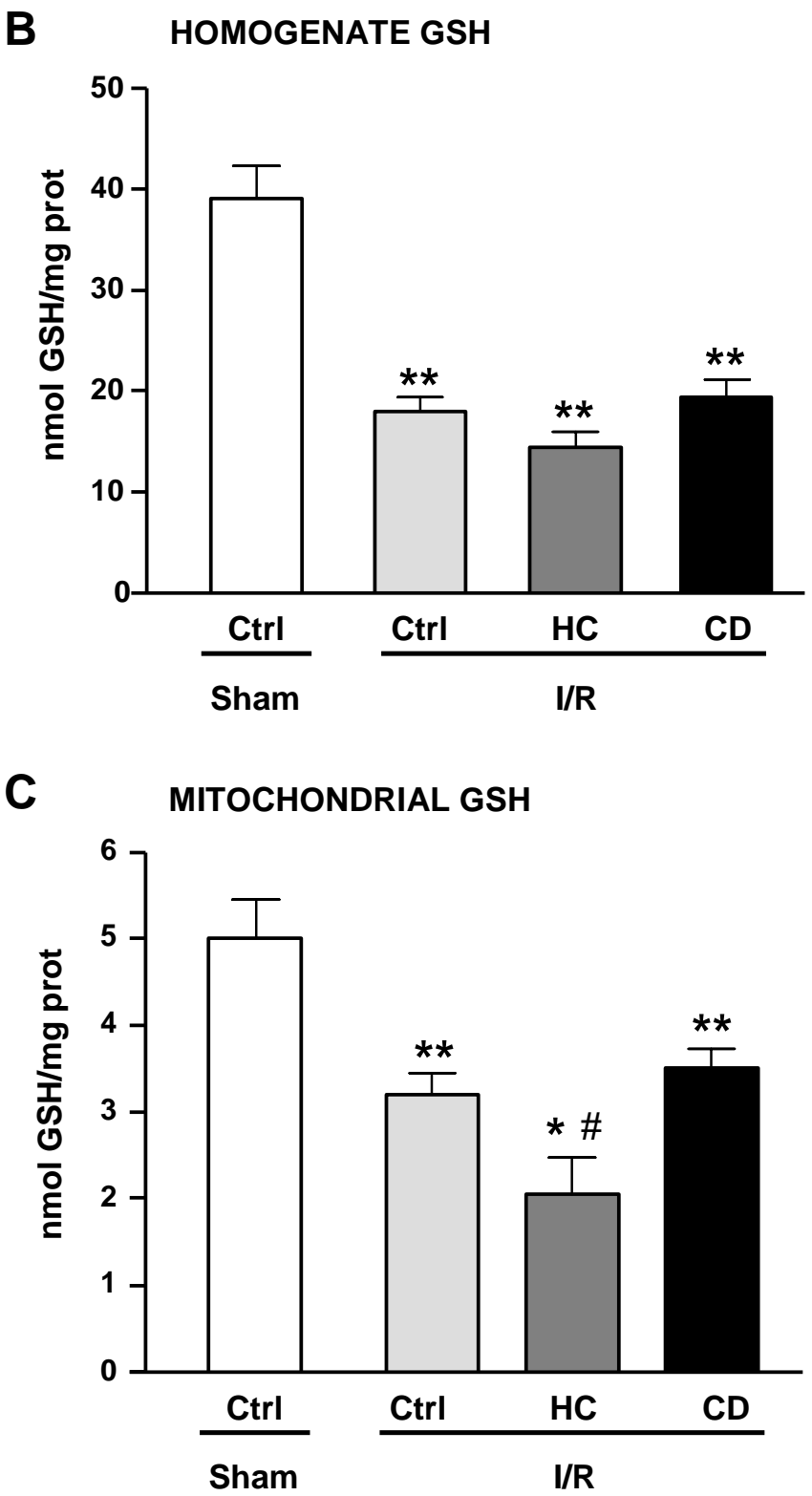
Figure 4

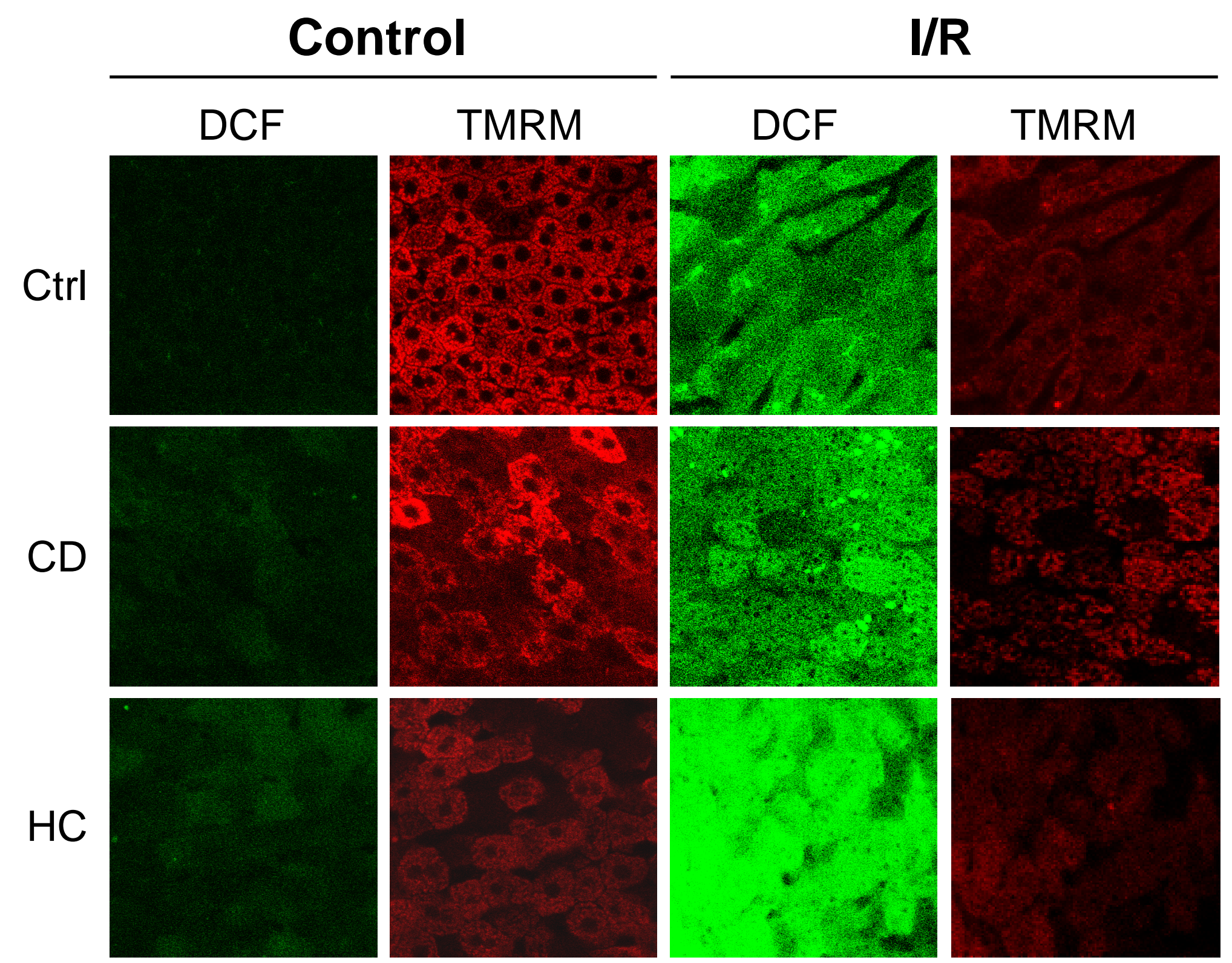


Figure 5

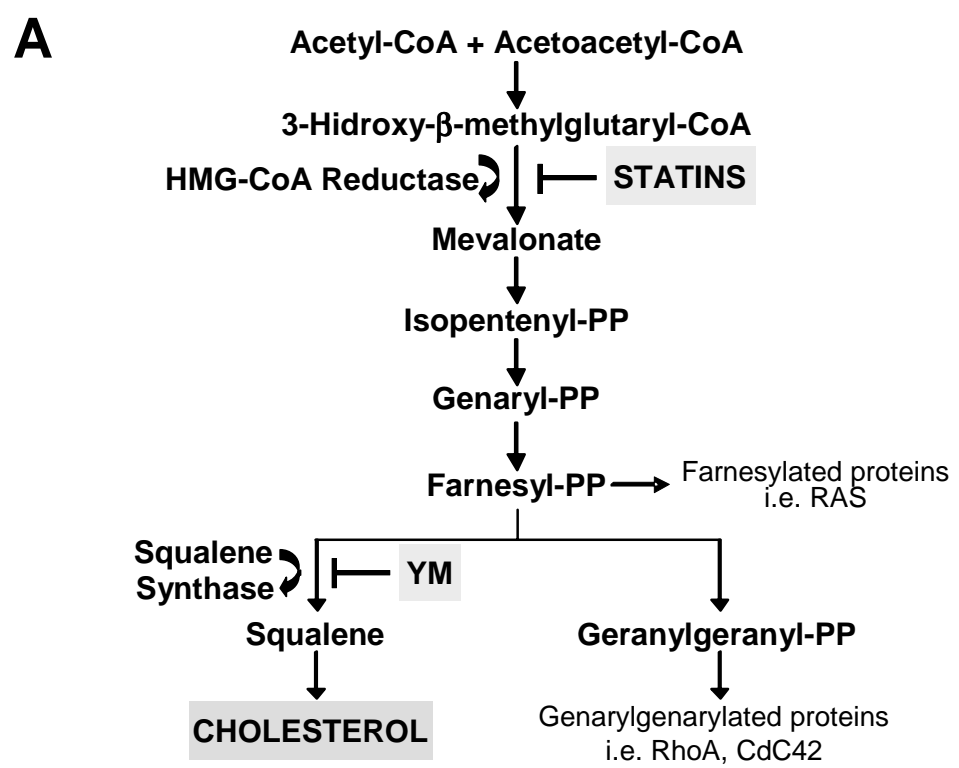

B

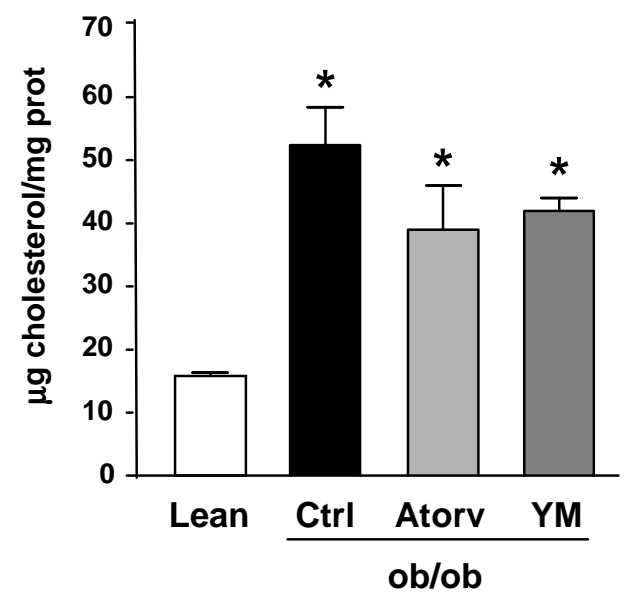

D

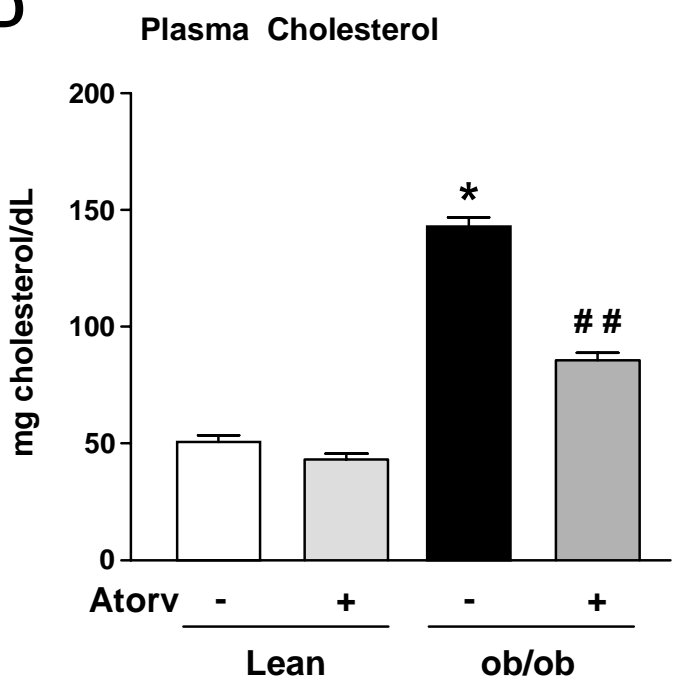

C

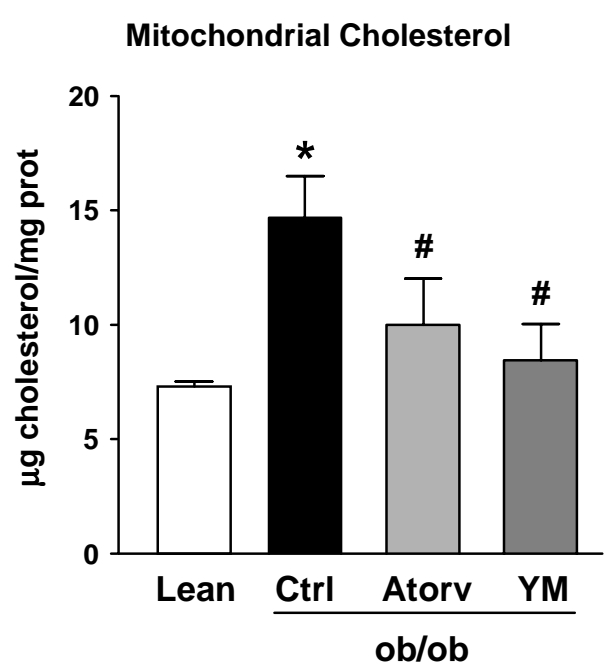

E

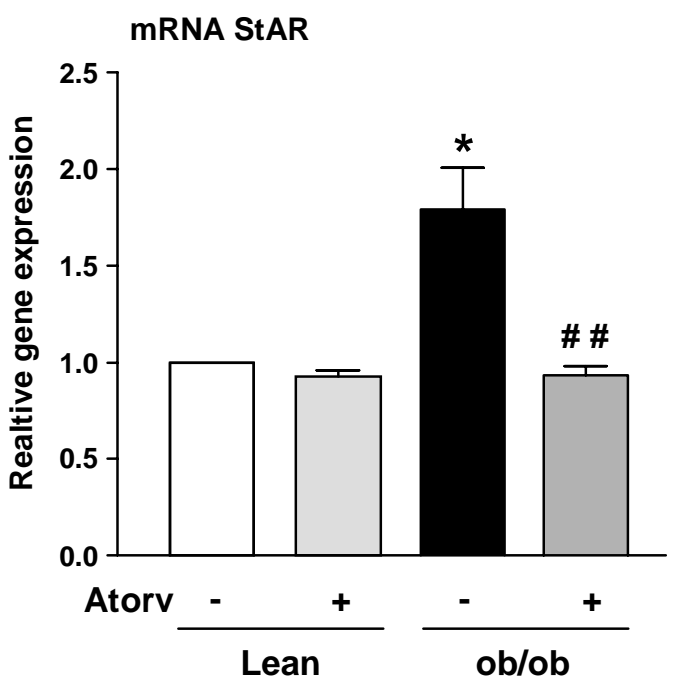


Figure 6

A

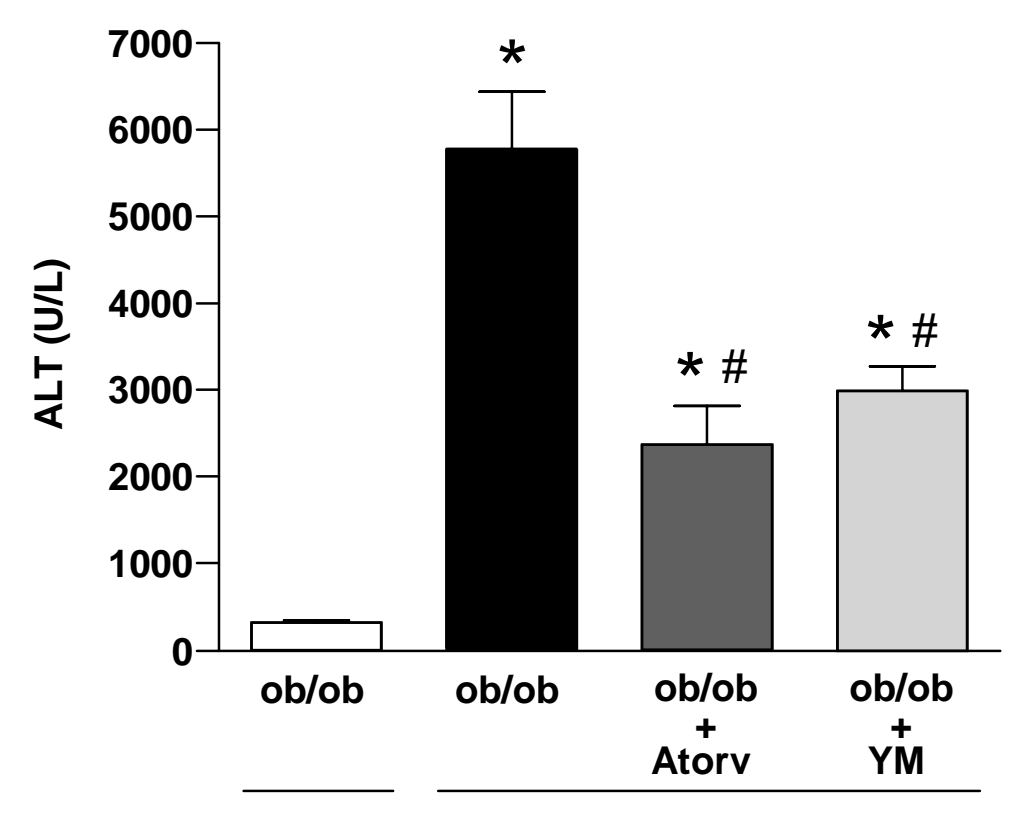

B

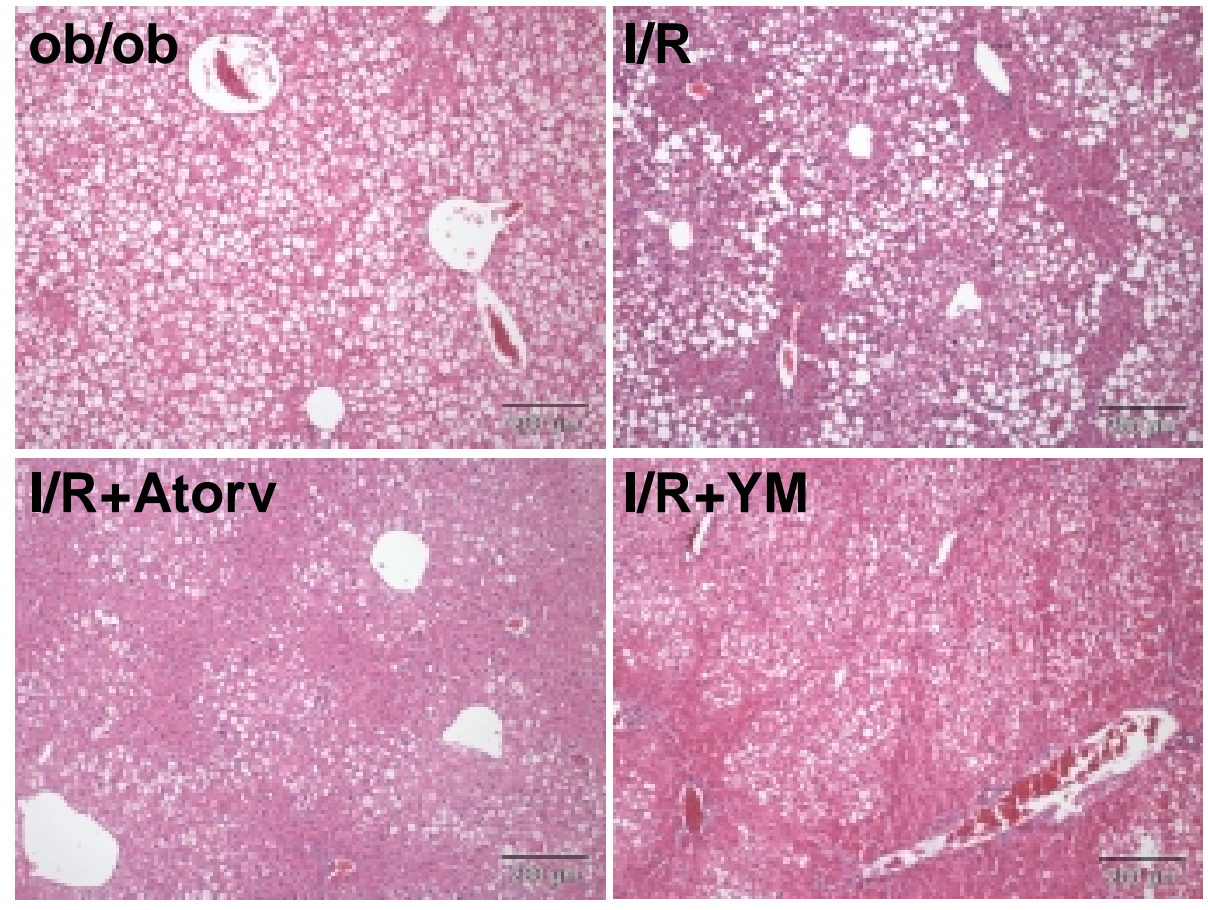


Figure 7

A

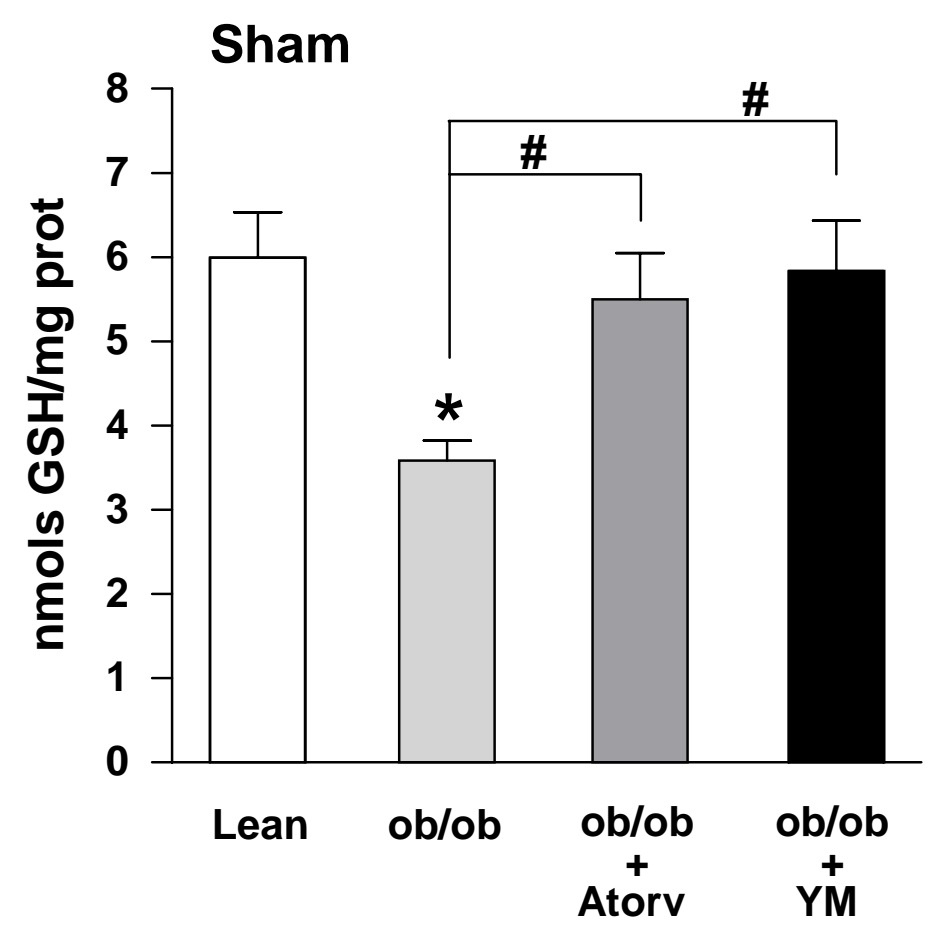

B

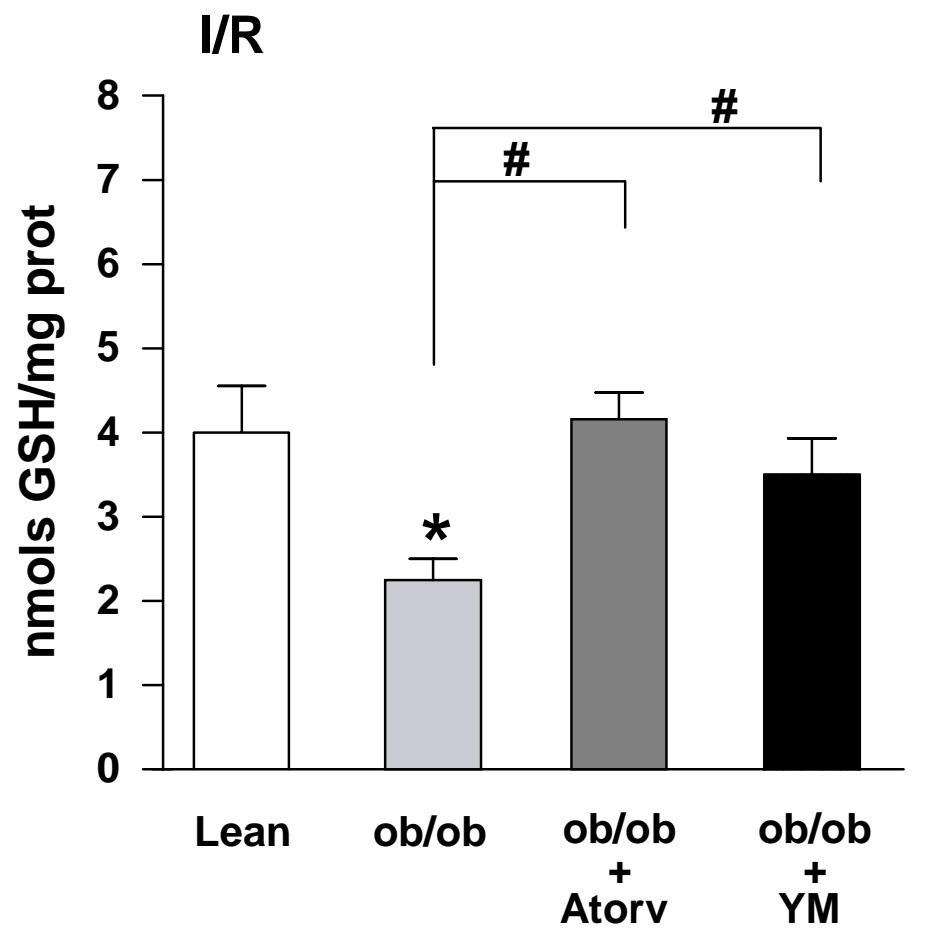


Figure 1 sup.

A

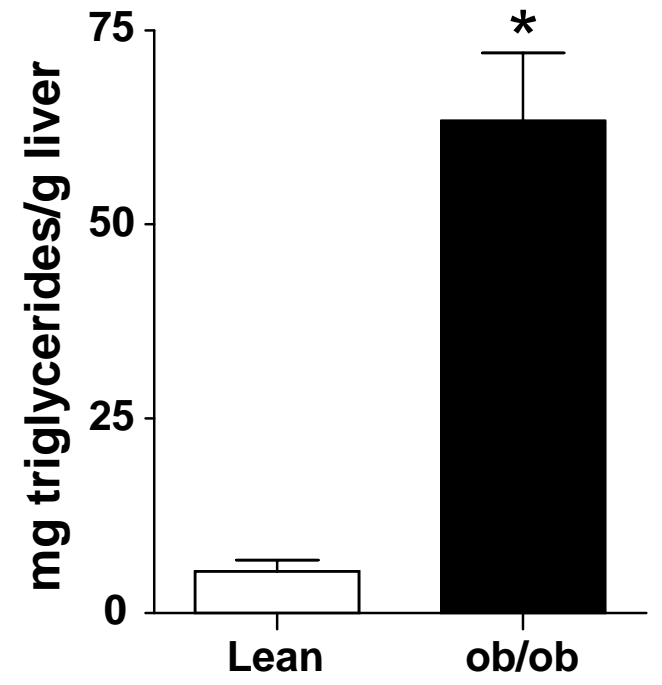

B

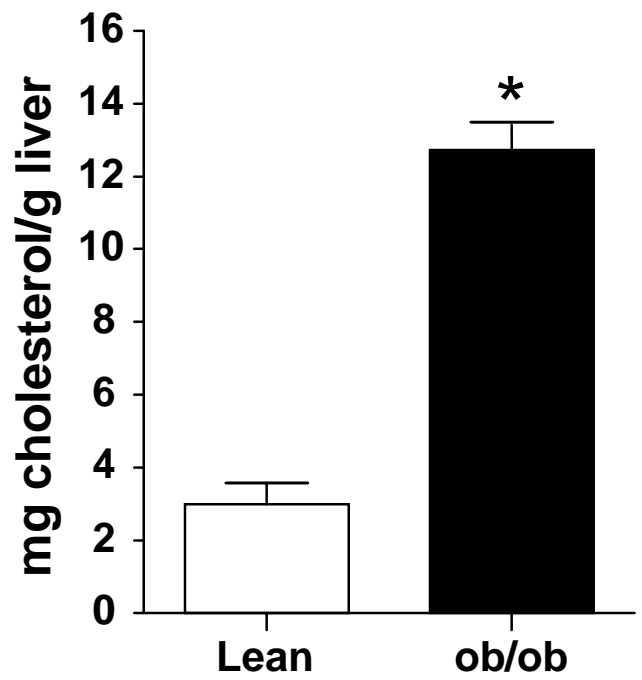

C

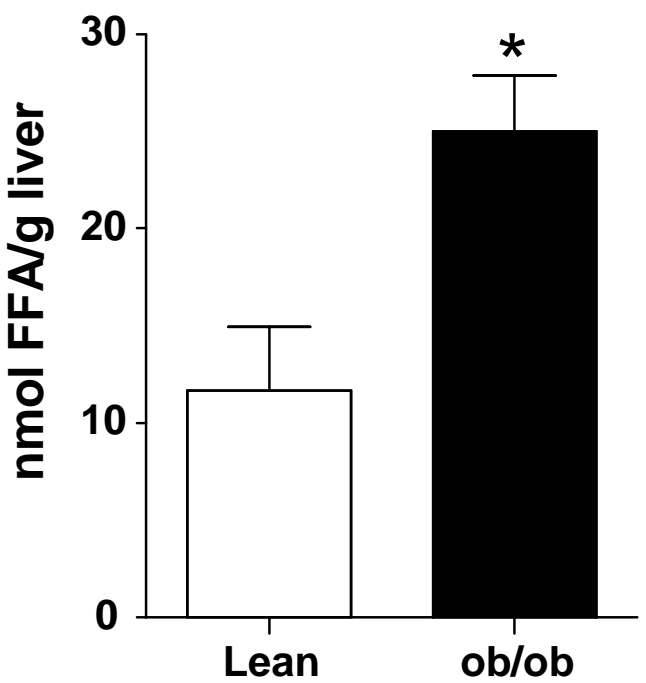


Figure 2 sup.

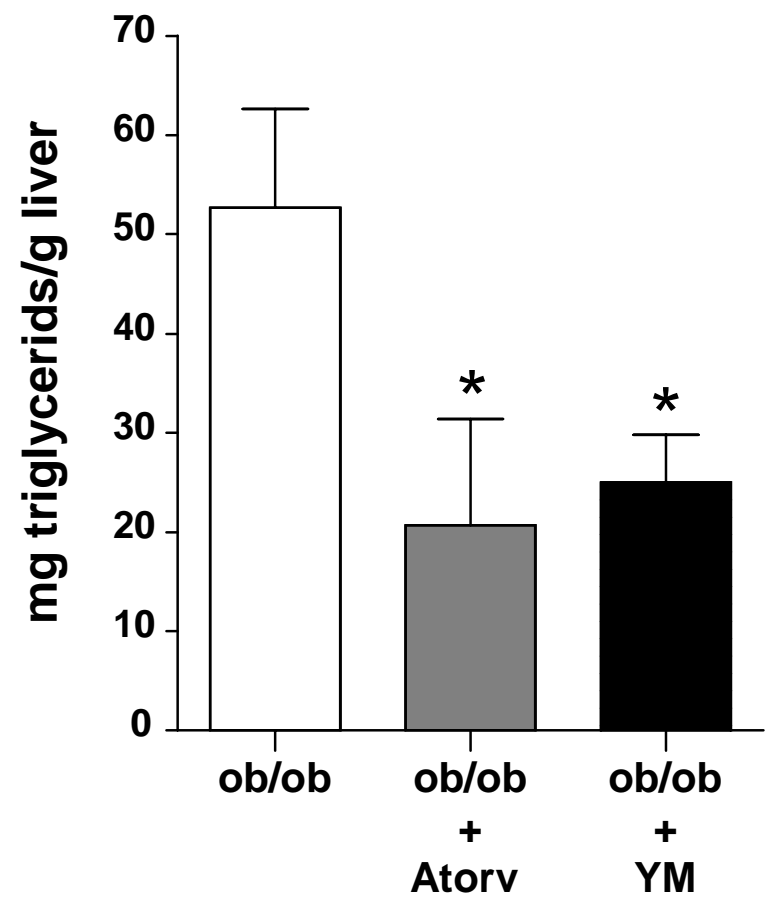


Figure 3 sup.

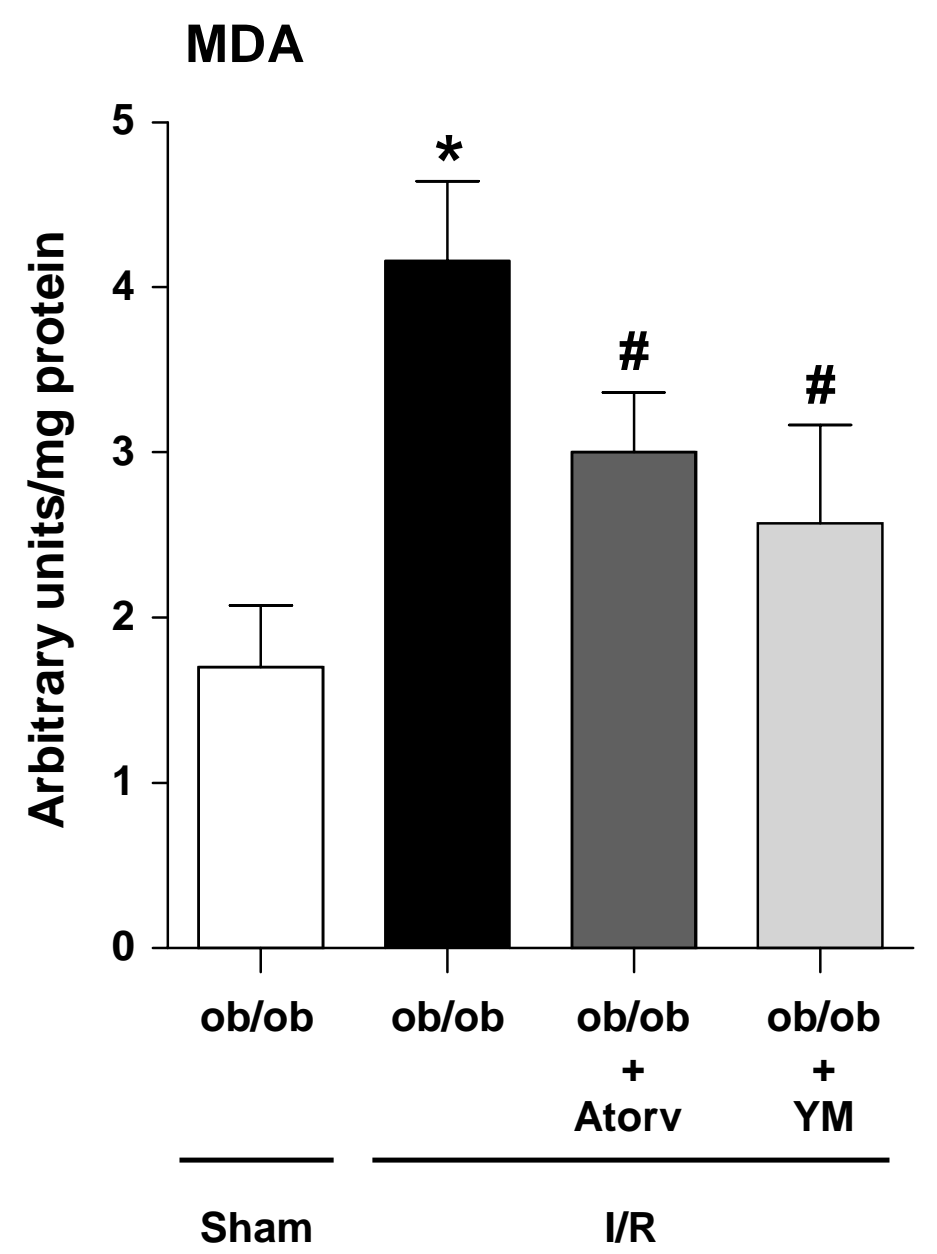

